\title{
Progress on Roman and Weakly Connected Roman Graphs
}

\author{
Joanna Raczek ${ }^{1, *}(\mathbb{D})$ and Rita Zuazua ${ }^{2}$ \\ 1 Department of Algorithms and Systems Modelling, Faculty of Electronics, Telecommunications and \\ Informatics, Gdańsk Tech, 80-233 Gdańsk, Poland \\ 2 Department of Mathematics, Faculty of Sciences, Universidad Nacional Autónoma de México, \\ Mexico City 4510, Mexico; ritazuazua@ciencias.unam.mx \\ * Correspondence: joanna.raczek@pg.edu.pl
}

check for

updates

Citation: Raczek, J.; Zuazua, R. Progress on Roman and Weakly Connected Roman Graphs. Mathematics 2021, 9, 1846. https:/ / doi.org/10.3390/math9161846

Academic Editor: Mikhail Goubko

Received: 30 June 2021

Accepted: 2 August 2021

Published: 5 August 2021

Publisher's Note: MDPI stays neutral with regard to jurisdictional claims in published maps and institutional affiliations.

\begin{abstract}
A graph $G$ for which $\gamma_{R}(G)=2 \gamma(G)$ is the Roman graph, and if $\gamma_{R}^{w c}(G)=2 \gamma_{w c}(G)$, then $G$ is the weakly connected Roman graph. In this paper, we show that the decision problem of whether a bipartite graph is Roman is a co-NP-hard problem. Next, we prove similar results for weakly connected Roman graphs. We also study Roman trees improving the result of M.A. Henning's A characterization of Roman trees, Discuss. Math. Graph Theory 22 (2002). Moreover, we give a characterization of weakly connected Roman trees.
\end{abstract}

Keywords: Roman domination number; weakly connected Roman domination number; weakly connected Roman graphs; NP completeness

MSC: 05C69; 05C05; 03D15; 05C76

\section{Introduction}

A Roman dominating function (RDF) on a graph $G=(V, E)$ was defined by Cockayne et al. in [1] as a function $f: V \rightarrow\{0,1,2\}$ such that every vertex $x$ with $f(x)=0$ is adjacent to at least one vertex $y$ for which $f(y)=2$. Let $V_{0}, V_{1}$, and $V_{2}$ be the sets of vertices such that they are assigned values 0,1 , and 2 , respectively, under $f$. Since there is one-to-one correspondence between the ordered triple $\left(V_{0}, V_{1}, V_{2}\right)$ and the function $f: V \rightarrow\{0,1,2\}$, we will write $f=\left(V_{0}, V_{1}, V_{2}\right)$ for short. The weight of $f$ is $w(f)=\sum_{v \in V} f(v)$, that is $w(f)=\left|V_{1}\right|+2\left|V_{2}\right|$. Similarly, for $S \subseteq V$, we define $f(S)=\sum_{v \in S} f(v)$. Hence, $w(f)=f(V)$. The Roman domination number, denoted $\gamma_{R}(G)$, is the minimum weight of an RDF in $G$. We define an RDF of weight $\gamma_{R}(G)$ as a $\gamma_{R}(G)$-function. Roman domination in graphs is of interest to many and has been studied, for example, in [1-3]. As it is made known in [4], the definition of a Roman dominating function was motivated by an article by Ian Stewart entitled "Defend the Roman Empire!" [5]. Each vertex in a graph represents a spot in the Roman Empire, and we want to defend this Empire from barbarians. A location (vertex $v$ ) is meant to be unsecured if no legions are currently stationed there (i.e., $f(v)=0)$ and secured otherwise (i.e., if $f(v) \in\{1,2\}$, where the number states for the number of legions in $v$ ). An unsecured vertex $v$ can be defended by an adjacent vertex $u$ with $f(u)=2$ by sending one legion from $u$ to $v$. A vertex $u$ with $f(u)=1$ cannot defend any unsecured vertex. These graph theory definitions are formed on the basis of the ideas of the Emperor Constantine the Great who wanted to modernize their army in the fourth century. Since it was expensive to maintain a vast army, the emperor wanted to support as few legions as possible while still defending the whole Roman Empire. A Roman dominating function of weight $\gamma_{R}(G)$ corresponds to such an optimal assignment of legions to locations.

In [6], the authors define and investigate the idea of strengthening the security of the Roman Empire by providing a safe way of communication between the legions while still having substantial costs of financing the legions as small as possible. Two legions at two different spots (secured vertices $u$ and $v$ ) can contact directly if there is at most one unsecured vertex between them, and the distance between $u$ and $v$ is at most 2 . Conversely, $u$ and 
$v$ can contact indirectly if there is a sequence of secured vertices $\left(u=u_{1}, u_{2}, \ldots, u_{k}=v\right)$ such that $u_{i}$ and $u_{i+1}$ can contact directly for $i=1,2, \ldots, k-1$. A graph is communicated if any two different secured vertices can contact directly or indirectly.

Following [7], we introduce some graph-related theoretical terminology. For a simple graph $G$, let $|V(G)|=n(G)$ be the order of $G$. The neighborhood $N_{G}(v)$ of a vertex $v \in V(G)$ is the set of all vertices adjacent to $v$ and the closed neighborhood is defined as $N_{G}[v]=N_{G}(u) \cup\{v\}$. If $D \subseteq V(G)$, then $N_{G}[D]=\bigcup_{v \in D} N_{G}[v]$. The degree $d_{G}(v)$ of $v$ is the number of edges incident with $v$ in $G$, so $d_{G}(v)=\left|N_{G}(v)\right|$. Let $L(G)$ be the set of all leaves of $G$, that is, the set of vertices of degree 1 . Next, $v$ is called a support vertex if $v$ is a neighbor of a leaf. Let $S(G)$ denote the set of all support vertices in $G$. A strong support vertex is a vertex adjacent to at least two leaves, while a vertex adjacent to exactly one leaf is a weak support vertex. A set $D \subseteq V(G)$ is a dominating set of $G$ if every vertex $v \in V(G)-D$ is adjacent to a vertex $u$ belonging to $D$. The minimum cardinality of a dominating set in $G$ is the domination number, denoted $\gamma(G)$.

In [1] is proved that for every graph, its Roman domination number is bounded from above by twice the domination number. Graphs $G$ with $\gamma_{R}(G)=2 \gamma(G)$, or just $\left(\gamma_{R}, 2 \gamma\right)$ graphs for short, are called Roman graphs. The following classes of graphs are established to be Roman [1]: $P_{3 k}, P_{3 k+2}$ for $k \geq 1, C_{3 k}, C_{3 k+2}$ for $k \geq 1, K_{m, n}$ for $\min \{m, n\} \neq 2$, any graph with $\Delta(G)=n-1$. Henning [3] characterized Roman trees and Xueliang et al. [8] characterized the Roman graphs in the following classes of graphs: circulant graphs, generalized Petersen graphs, and the Cartesian product of cycles.

From now on, we assume $G$ to be connected. The subgraph weakly induced by a set $D \subseteq V(G)$ is the graph $\langle D\rangle_{w}=\left(N[D], E_{w}\right)$, where $E_{w}$ is the set of all edges of $G$ that have at least one end vertex in $D$. A set $D \subseteq V(G)$ is a weakly connected dominating set (WCDS) of $G$ if $D$ is both a dominating set, and $\langle D\rangle_{w}$ is connected. The weakly connected domination number of $G$, denoted $\gamma_{w c}(G)$, is the minimum cardinality of a WCDS of G. A minimum WCDS of a graph $G$ is called a $\gamma_{w c}(G)$-set. The weakly connected domination number was introduced in [9] and studied, for example, in [10-12].

The function $f=\left(V_{0}, V_{1}, V_{2}\right)$ is called a weakly connected Roman dominating function in $G$ (WCRDF) if $f$ is an RDF, and the subgraph $\left\langle V_{1} \cup V_{2}\right\rangle_{w}$ weakly induced by $V_{1} \cup V_{2}$ is connected in $G$. The weakly connected Roman domination number, denoted $\gamma_{R}^{w c}(G)$, is the minimum weight of a WCRDF in $G$, that is, $\gamma_{R}^{w c}(G)=\min \{w(f): f$ is a WCRDF in $G\}$. A WCRDF of weight $\gamma_{R}^{w c}(G)$ is called a $\gamma_{R}^{w c}(G)$-function. These terms were first introduced and motivated in [6].

In [6], it is proven as follows:

Proposition 1 ([6]). If $G$ is a connected graph, then

$$
\gamma_{w c}(G) \leq \gamma_{R}^{w c}(G) \leq 2 \gamma_{w c}(G) .
$$

The authors of [6] also describe all graphs for which the lower bound is attained.

Proposition 2 ([6]). For any connected graph $G$ of order $n, \gamma_{w c}(G)=\gamma_{R}^{w c}(G)$ if and only if $G=K_{1}$.

Graphs $G$ for which $\gamma_{R}^{w c}(G)=2 \gamma_{w c}(G)$ are the weakly connected Roman graphs.

In this paper, we show that the decision problem of whether a bipartite graph is Roman is a co-NP-hard problem and we also study Roman trees on this basis [3]. We also show that the decision problem of whether a bipartite graph is a weakly connected Roman is a co-NP-hard problem. In addition, we give a characterization of weakly connected Roman trees.

\section{Complexity Results}

In this section, we state the computational complexity of decision problems for Roman and weakly connected Roman bipartite graphs. 
The three-satisfiability (3SAT) problem is as follows [13]:

INSTANCE: A literal is a logical variable or its negation. We are given a Boolean expression $E$ in conjunctive normal form (CNF), that is, the conjunction of clauses, each of which is the disjunction of three distinct literals.

QUESTION: Is E satisfiable?

Theorem 1. It is co-NP-hard to determine if a given graph $G$ is Roman even for bipartite graphs.

Proof. We describe a polynomial reduction from 3SAT to the considered problem. The 3SAT problem was proven to be NP-complete in [13]. Without loss of generality, we can assume that the formula in 3SAT contains the instance of any logical variable $u$ and its negation $u^{\prime}$, because in the other case, all clauses containing the variable $u$ (or $u^{\prime}$ ) are satisfied by assigning true (false) to $u$. We also assume that the formula contains at least two clauses (otherwise the answer is trivial).

For an instance $E$ of 3SAT, denote the set of variables of $E$ by $U=\left\{u_{1}, u_{2}, \ldots, u_{n}\right\}$ and the set of clauses by $C=\left\{c_{1}, c_{2}, \ldots, c_{m}\right\}$. Based on $E$, we construct a graph $G$ whose order and size are polynomially bounded from above in terms of $n$ and $m$, and such that the formula $E$ is satisfiable if and only if $\gamma_{R}(G)<\gamma(G)$. At first, for each variable $u_{i}$ construct a copy of $G\left(u_{i}\right)$ of the graph in Figure 1 . Vertex $u_{i}$ corresponds to literal $u_{i}$, while vertex $u_{i}^{\prime}$ - to the negation of $u_{i}, \neg u_{i}$. Notice that $G\left(u_{i}\right)$ is a bipartite graph.



Figure 1. The graph $G\left(u_{i}\right)$.

For each one clause $C_{j}$, we create a clause vertex $c_{j}$ and if $C_{j}$ contains literals $x, y$, and $z$, we create the three edges $c_{j} x, c_{j} y$, and $c_{j} z$. If, for example, $C_{1}=\neg u_{1} \vee u_{2} \vee u_{3}$, then these edges are $c_{1} u_{1}^{\prime}, c_{1} u_{2}$, and $c_{1} u_{3}$. Additionally, we add two vertices, $a$ and $b$, and next we add the edges $a c_{j}$ for $j=1,2 \ldots, m$, and $b u_{i}, b u_{i}^{\prime}$ for $i=1,2, \ldots, n$, as shown in Figure 2. This accomplishes the depiction of $G$. It is easy to see that $G$ is bipartite.

Observe that for each graph, $G\left(u_{i}\right), u_{i}$, or $u_{i}^{\prime}$ belongs to every minimum dominating set of $G$. Since $a$ is adjacent to each clause vertex and $b$ is adjacent to each variable vertex and its negation, it is clear that $\gamma(G)=n+2$, and hence, $\gamma_{R}(G) \leq 2 n+4$. Therefore, it remains to be proven that $E$ is satisfiable if and only if $\gamma_{R}(G)<2 n+4$.

First, we assume that $E$ is satisfiable and consider a satisfying truth assignment. Let $f$ be a function assigning 2 to each true variable vertex (or its negation) and $b$, let $f(a)=1$ and let $f$ assign 0 to every other vertex of $G$. Since $E$ is satisfiable, each clause vertex $c_{j}$ is adjacent to a variable vertex (or its negation) $u$ with $f(u)=2$. Similarly, each vertex of $C\left(u_{i}\right)$ has in its closed neighborhood a vertex $u$ with $f(u)=2$. Therefore, $f$ is a Roman dominating function of $G$. Since $|f|=2 n+3, \gamma_{R}(G)<2 \gamma(G)$, implying that $G$ is not a Roman graph.

Next, we assume that $E$ is not satisfiable. Then, for each truth assignment for some $j \in 1,2, \ldots, m$ each literal of the clause $C_{j}$ is false. Let $f$ be a function assigning 2 to each true variable vertex (or its negation) and $b$. If $f(a)=1$ and $f$ assigns 0 to every other vertex of $G$, then $f\left(c_{j}\right)=0$ and no vertex if the closed neighborhood of $c_{j}$ has the $f$ function value equal to 2 . Therefore, in this case, let $f(a)=2$ and let $f$ assign 0 to every other vertex of $G$. Then, $|f|=2 n+4$, and $G$ is a Roman graph. This completes the proof. 


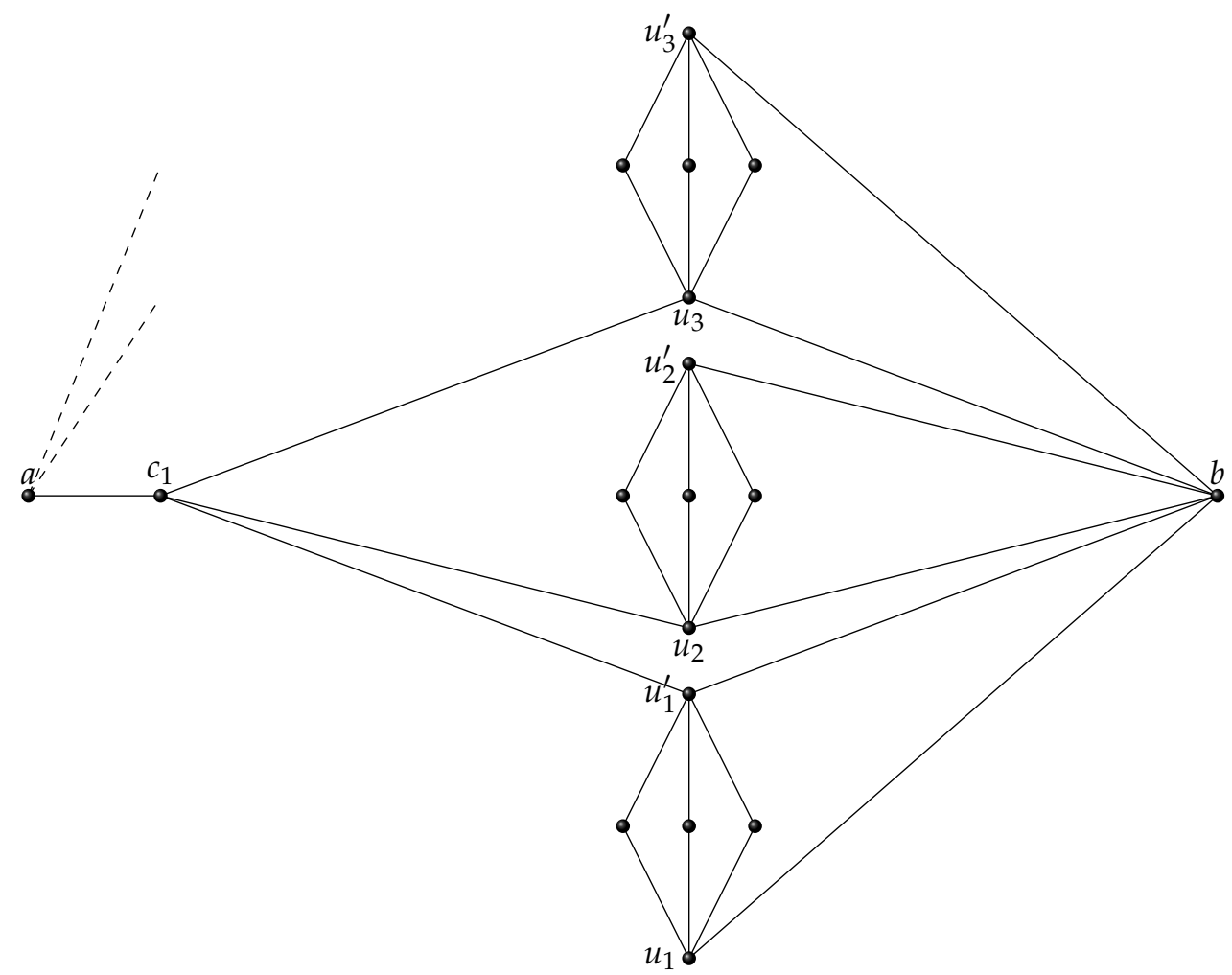

Figure 2. The edges between $G\left(C_{1}\right)$ and $G\left(u_{1}\right) \cup G\left(u_{2}\right) \cup G\left(u_{3}\right)$ for the clause $C_{1}=\neg u_{1} \vee u_{2} \vee u_{3}$.

Theorem 2. It is co-NP-hard to determine if a given graph $G$ is a weakly connected Roman even for bipartite graphs.

Proof. The construction and the proof are analogous to the previous proof, however, we add one additional edge to the graph $G$, namely, $a w_{1}^{1}$, to provide the minimum dominating set of $G$ to be weakly connected even if no clause has a true literal.

\section{Roman Trees}

Henning, in [3], has constructively characterized $\left(\gamma_{R}, 2 \gamma\right)$-trees as a family $\mathcal{T}$ of all rooted trees that can be obtained from a star $K_{1, r}$ for $r \geq 1$ by applying recursively three operations. Two of those operations attach new small tree structures to the vertex $v$ of the existing tree from $\mathcal{T}$, where $v \in V_{S}(T)$. The set $V_{S}(T)$ is defined as follows: $V_{S}(T)=\left\{v \in V(T): v \in S(T)\right.$ and $\left.\gamma_{R}(T-v) \geq \gamma_{R}(T)\right\}$. For details of the construction please check [3].

However, the characterization given in [3] does not include all $\left(\gamma_{R}, 2 \gamma\right)$-trees. For example, a tree $T$ presented in Figure 3 is a Roman tree since $\gamma_{R}(T)=2 \gamma(T)=8$, but it is not possible to obtain $T$ using the characterization given in [3].

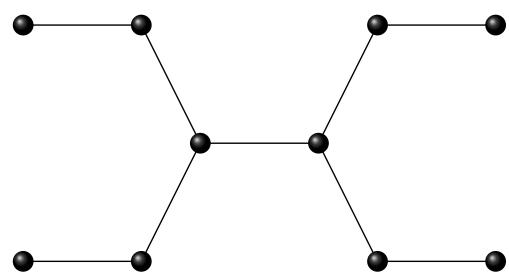

Figure 3. A $\left(\gamma_{R}, 2 \gamma\right)$-tree not obtained by the original characterization [3], see also [14].

To fully characterize all Roman trees, the definition of $V_{S}(T)$ needs to be changed as follows

$$
V_{S}(T)=\left\{v \in V(T): \gamma_{R}(T-v) \geq \gamma_{R}(T)\right\}
$$


Then more trees are members of the family $\mathcal{T}$. Despite the change in the definition of $V_{S}(T)$, the proofs in the original work of Henning [3] are correct for the new version of $V_{S}(T)$ because of the assumption that a vertex $v \in V_{S}(T)$ is a support vertex in the proofs of [3] and is not used.

\section{Weakly Connected Roman Trees}

In this section, we describe all trees $T$ for which $\gamma_{R}^{w c}(T)=2 \gamma_{w c}(T)$.

Let $T$ be a tree. A vertex $v$ belongs to the set $A \subset V(T)$ if and only if $v$ is of degree at least 2 ( $v$ is not a leaf) and $N(v) \subseteq S(T)$, that is, $A=\left\{v \in V(T) \mid d_{T}(v) \geq 2\right.$ and $N(v) \subseteq$ $S(T)\}$.

Let $v$ be a weak support vertex of $T$. Depending on its neighborhood, we classify it as type-1, type-2, or type-1a weak support vertex. The weak support vertex $v$ is

- $\quad$ Type- 1 , if $v$ is adjacent to exactly one vertex of $A$, that is $|N(v) \cap A|=1$;

- $\quad$ Type-2, if $v$ is adjacent to more that one vertex of $A$, that is $|N(v) \cap A|>1$;

- $\quad$ Type-1a, if $v$ is type- 1 and $v$ is adjacent to a strong support vertex or to a weak support vertex type-2.

Definition 1. Let $\mathcal{T}$ be the family of all trees such that

1. $P_{1}, P_{2} \in \mathcal{T}$;

2. $V(T)=L(T) \cup S(T) \cup A$;

3. If $v$ is a weak support vertex, then $v$ is adjacent to a vertex of $A$;

4. If $v \in A$, then $v$ is adjacent to at most two weak support vertices of type-1;

5. If $v \in A$, then $v$ is adjacent to at most one weak support vertex of type-1a.

Property 1. Let $T$ be a tree such that $\gamma_{R}^{w c}(T)=2 \gamma_{w c}(T)$. Then, each vertex of a minimum weakly connected dominating set of $T$ is either a leaf or a support vertex.

Proof. Suppose that a vertex $v$ belongs to a minimum weakly connected dominating set of $T$, say $D_{w c}$, and $v$ is neither a support vertex nor a leaf. Then each neighbor of $v$ is adjacent to a vertex of $D_{w c}-\{v\}$ or belongs to $D_{w c}$. However in this situation, $\left(V(T)-D_{w c}, v, D_{w c}-\{v\}\right)$ is a weakly connected Roman dominating function of $T$ of weight smaller than $2 \gamma_{w c}(T)$, a contradiction.

Lemma 1. Let $T$ be a tree. If $\gamma_{R}^{w c}(T)=2 \gamma_{w c}(T)$, then $T$ belongs to the family $\mathcal{T}$.

Proof. The result is obvious for $P_{1}, P_{2}$, and for trees of diameter 2 . Thus, in what follows, we focus on trees of diameter at least 3. By Claim 1, $V(T)=L(T) \cup S(T) \cup A$, which is condition 2 of Definition 1. Moreover, if $\gamma_{R}^{w c}(T)=2 \gamma_{w c}(T)$, then $S(T)$ is a minimum weakly connected dominating set of $T$ and a weakly connected Roman dominating function assigning 2 to each support vertex and 0 to every other vertex, namely, $(V(T)-S(T), \varnothing, S(T))$, is a $\gamma_{R}^{w c}(T)$-function.

Assume that $\gamma_{R}^{w c}(T)=2 \gamma_{w c}(T)$ and $v$ is a weak support vertex such that $v$ is not adjacent to a vertex of $A$. Then one neighbor of $v$ is a leaf, say $u$, and every other neighbor is a support vertex. Moreover, $f=(V-S(T), \varnothing, S(T))$ is a $\gamma_{R}^{w c}(T)$-function. However, $((V(T)-S(T)-\{u\}) \cup\{v\},\{u\}, S(T)-\{v\})$ is a weakly connected Roman dominating function of $T$ of weight smaller than $\gamma_{R}^{w c}(T)$, a contradiction. Therefore, if $\gamma_{R}^{w c}(T)=2 \gamma_{w c}(T)$, then each weak support vertex is adjacent to a vertex of $A$, which is condition 3 of Definition 1.

Assume now that $\gamma_{R}^{w c}(T)=2 \gamma_{w c}(T)$ and $v \in A$ is adjacent to at least three weak support vertices of type- 1 denoted $u_{1}, u_{2}, u_{3}$. Denote by $w_{1}, w_{2}, w_{3}$ the leaves adjacent to $u_{1}, u_{2}, u_{3}$, respectively. Again $f=(V(T)-S(T), \varnothing, S(T))$ is a $\gamma_{R}^{w c}(T)$-function. However, $\left(\left((V(T)-S(T))-\left\{v, w_{1}, w_{2}, w_{3}\right\}\right) \cup\left\{u_{1}, u_{2}, u_{3}\right\},\left\{w_{1}, w_{2}, w_{3}\right\},(S(T) \cup\{v\})-\left\{u_{1}, u_{2}, u_{3}\right\}\right)$ is a weakly connected Roman dominating function of $T$ of weight smaller than $\gamma_{R}^{w c}(T)$, a 
contradiction. Therefore, if $\gamma_{R}^{w c}(T)=2 \gamma_{w c}(T)$, then each vertex of $A$ is adjacent to at most two weak support vertices of type-1, which is condition 4 of Definition 1.

Lastly, assume that $\gamma_{R}^{w c}(T)=2 \gamma_{w c}(T)$ and $v \in A$ is adjacent to at least two weak support vertices of type-1a denoted $u_{1}, u_{2}$. Denote by $w_{1}, w_{2}$ the leaves adjacent to $u_{1}, u_{2}$, respectively. Clearly, $f=(V(T)-S(T), \varnothing, S(T))$ is a $\gamma_{R}^{w c}(T)$-function. Since $u_{1}, u_{2}$ are type-1a, $\left.\left(\left(V(T)-S(T)-\left\{v, w_{1}, w_{2}\right\}\right) \cup\left\{u_{1}, u_{2}\right\},\left\{v, w_{1}, w_{2}\right\}, S T\right)-\left\{u_{1}, u_{2}\right\}\right)$ is a weakly connected Roman dominating function of $T$ of weight smaller than $\gamma_{R}^{w c c}(T)$, a contradiction. Therefore, if $\gamma_{R}^{w c c}(T)=2 \gamma_{w c}(T)$, then each vertex of $A$ is adjacent to at most one weak support vertex of type-1a, which is condition 5 of Definition 1 .

Lemma 2. Let $T$ be a tree. If $T$ belongs to the family $\mathcal{T}$, then $\gamma_{R}^{w c}(T)=2 \gamma_{w c}(T)$.

Proof. Suppose that $T \in \mathcal{T}$, we will prove, by induction on the number of vertices of $T$, that $\gamma_{R}^{w c}(T)=2 \gamma_{w c}(T)$.

Let $P=\left(v_{0}, v_{1}, v_{2}, \ldots, v_{k}\right)$ be a longest path in $T$.

If $k \leq 4$ and $T \in \mathcal{T}$, then clearly $\gamma_{R}^{w c}(T)=2 \gamma_{w c}(T)$. Hence, assume $k \geq 5$ and $n(T) \geq 6$.

If $d\left(v_{2}\right)=d\left(v_{3}\right)=2$, then $v_{2} \notin L(T) \cup S(T) \cup A$, a contradiction with condition 2 of

Definition 1. Therefore, we can consider the following cases.

- Assume $d\left(v_{2}\right)=2, v_{2} \in A$ and $d\left(v_{3}\right)>2$. In this situation, $v_{1}, v_{3}$ are support vertices. Let $T^{\prime}$ be the connected component of $T-v_{1} v_{2}$ containing $v_{2}$. Since $T \in \mathcal{T}, T^{\prime}$ also fulfills all conditions of Definition 1 to be in $\mathcal{T}$. Hence, by the induction hypothesis, $\gamma_{R}^{w c}\left(T^{\prime}\right)=2 \gamma_{w c}\left(T^{\prime}\right)$.

Moreover, each $\gamma_{w c}\left(T^{\prime}\right)$-set can be extended to a weakly connected dominating set of $T$ by adding to it $v_{1}$, so $\gamma_{w c}(T) \leq \gamma_{w c}\left(T^{\prime}\right)+1$. Let $f=\left(V_{0}, V_{1}, V_{2}\right)$ be a $\gamma_{R}^{w c}(T)$-function. Without loss of generality, we may assume that $v_{1}, v_{3} \in V_{2}$, and $v_{2}$ as well as each leaf adjacent to $v_{1}$ belong to $V_{0}$. Then $f$ restricted on $T^{\prime}$ is a WCRDF, and therefore, $\gamma_{R}^{w c}\left(T^{\prime}\right) \leq \gamma_{R}^{w c}(T)-2$. Hence,

$$
2 \gamma_{w c}(T) \leq 2 \gamma_{w c}\left(T^{\prime}\right)+2=\gamma_{R}^{w c}\left(T^{\prime}\right)+2 \leq \gamma_{R}^{w c}(T)
$$

implying that $\gamma_{R}^{w c}(T)=2 \gamma_{w c}(T)$.

- Suppose $d\left(v_{2}\right)>2$ and $v_{2}$ is not a support vertex. Then, $v_{2}$ is adjacent to at least two support vertices and since $T \in \mathcal{T}$, by Definition $1 v_{2}$ is adjacent to at most two weak support vertices of type-1. Let $T^{\prime}$ be the connected component of $T-v_{1} v_{2}$ containing $v_{2}$. Since $T \in \mathcal{T}$, in this situation, $T^{\prime}$ also fulfills all conditions of Definition 1 to be in $\mathcal{T}$. Hence, by the induction hypothesis, $\gamma_{R}^{w c}\left(T^{\prime}\right)=2 \gamma_{w c}\left(T^{\prime}\right)$. Moreover, each $\gamma_{w c}\left(T^{\prime}\right)$-set can be extended to a weakly connected dominating set of $T$ by adding to it $v_{1}$, so $\gamma_{w c}(T) \leq \gamma_{w c}\left(T^{\prime}\right)+1$. Let $f=\left(V_{0}, V_{1}, V_{2}\right)$ be a $\gamma_{R}^{w c}(T)$-function. Without loss of generality, we may assume that each support vertex adjacent to $v_{2}$ is in $V_{2}$, and $v_{2}$, as well as each leaf adjacent to $v_{1}$ belong to $V_{0}$. Then, $f$ restricted on $T^{\prime}$ is a WCRDF, and therefore, $\gamma_{R}^{w c}\left(T^{\prime}\right) \leq \gamma_{R}^{w c}(T)-2$. Hence,

$$
2 \gamma_{w c}(T) \leq 2 \gamma_{w c}\left(T^{\prime}\right)+2=\gamma_{R}^{w c}\left(T^{\prime}\right)+2 \leq \gamma_{R}^{w c}(T)
$$

implying that $\gamma_{R}^{w c}(T)=2 \gamma_{w c}(T)$.

- $\quad$ Suppose $d\left(v_{2}\right)>2$ and $v_{2}$ is a support vertex. Then, $v_{1}$ is a strong support vertex and $d\left(v_{1}\right) \geq 3$. If $v_{2}$ is a weak support vertex, then $v_{3} \in A$. Let $T^{\prime}$ be the connected component of $T-v_{1} v_{2}$ containing $v_{2}$. Since $T \in \mathcal{T}$, in this situation, $T^{\prime}$ also fulfills all conditions of Definition 1 to be in $\mathcal{T}$. Hence, by the induction hypothesis, $\gamma_{R}^{w c}\left(T^{\prime}\right)=2 \gamma_{w c}\left(T^{\prime}\right)$. Moreover, each $\gamma_{w c}\left(T^{\prime}\right)$-set can be extended to a weakly connected dominating set of $T$ by adding to it $v_{1}$, so $\gamma_{w c}(T) \leq \gamma_{w c}\left(T^{\prime}\right)+1$. Let $f=\left(V_{0}, V_{1}, V_{2}\right)$ be a $\gamma_{R}^{w c}(T)$-function. Without loss of generality, we may assume that 
$v_{1}, v_{2}$ are in $V_{2}$ and each leaf adjacent to $v_{1}$ or $v_{2}$ belongs to $V_{0}$. Then $f$ restricted on $T^{\prime}$ is a WCRDF, and therefore, $\gamma_{R}^{w c}\left(T^{\prime}\right) \leq \gamma_{R}^{w c}(T)-2$. Hence,

$$
2 \gamma_{w c}(T) \leq 2 \gamma_{w c}\left(T^{\prime}\right)+2=\gamma_{R}^{w c}\left(T^{\prime}\right)+2 \leq \gamma_{R}^{w c}(T)
$$

implying that $\gamma_{R}^{w c}(T)=2 \gamma_{w c}(T)$.

By Lemmas 1 and 2 we obtain the next theorem.

Theorem 3. Let $T$ be a tree. Then,

$$
\gamma_{R}^{w c}(T)=2 \gamma_{w c}(T)
$$

if and only if $T$ belongs to the family $\mathcal{T}$.

Since the definition of the family $\mathcal{T}$ considers only closed neighborhoods of the vertices of a tree, the weakly connected Roman trees can be recognized in polynomial time.

The tree in Figure 4 is an example of a graph belonging to the family $\mathcal{T}$.

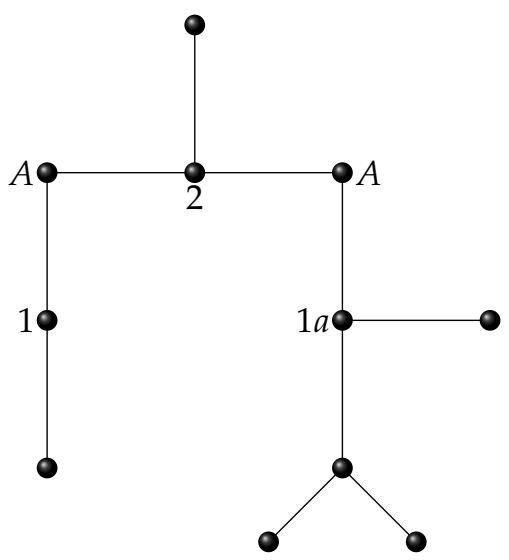

Figure 4. The tree in $\mathcal{T}$. Markings denote the type of a weak support vertex

Author Contributions: Conceptualization, R.Z.; methodology, J.R. and R.Z.; formal analysis, J.R. and R.Z.; investigation, R.Z. and J.R.; resources, J.R. and R.Z.; writing-original draft preparation, J.R.; writing-review and editing, J.R. All authors have read and agreed to the published version of the manuscript.

Funding: The second author thanks the financial support of travel costs received from Grant UNAMPAPIIT IN-117219.

Conflicts of Interest: The authors declare no conflict of interest.

\section{Abbreviations}

The following abbreviations are used in this manuscript:

WCDF Weakly connected dominating set

WCRDF Weakly connected Roman dominating function

3SAT The three-satisfiability problem

CNF Conjunctive normal form

\section{References}

1. Cockayne, E.J.; Dreyer, P.A.; Hedetniemi, S.M.; Hedetniemi, S.T. Roman domination in graphs. Discret. Math. 2004, 278, 11-22. [CrossRef]

2. Dreyer, P. Defending the Roman Empire. Ph.D. Thesis, Rutgers University, New Brunswick, NJ, USA, 2000.

3. Henning, M.A. A characterization of Roman trees. Discuss. Math. Graph Theory 2002, 22, 225-234. [CrossRef] 
4. Henning, M.A.; Hedetniemi, S.T. Defending the Roman Empire-A new strategy. Discret. Math. 2003, 266, 239-251. [CrossRef]

5. Stewart, I. Defend the Roman Empire! Sci. Am. 1999, 281, 136-139. [CrossRef]

6. Raczek, J.; Cyman, J. Weakly connected Roman domination in graphs. Discret. App. Math. 2019, 267, 151-159. [CrossRef]

7. Haynes, T.W.; Hedetniemi, S.T.; Slater, P.J. Fundamentals of Domination in Graphs; Marcel Dekker Inc.: New York, NY, USA, 1998.

8. Xueliang, F.; Yuansheng, Y.; Baoqi, J. Roman domination in regular graphs. Discret. Math. 2009, 309, 1528-1537. [CrossRef]

9. Dunbar, J.E.; Grossman, J.W.; Hattingh, J.H.; Hedetniemi, S.T.; McRae, A.A. On weakly connected domination in graphs. Discret. Math. 1997, 167-168, 261-269. [CrossRef]

10. Domke, G.S.; Hattingh, J.H.; Marcus, L.R. On weakly connected domination in graphs II. Discret. Math. 2005, 305, 112-122. [CrossRef]

11. Lemańska, M.; Patyk, A. Weakly connected domination critical graphs. Opusc. Math. 2008, 28, 325-330.

12. Swaminathan, V. Weakly Connected Domination in Graphs, Electronic Notes. Discret. Math. 2009, 33, 67-73.

13. Garey, M.R.; Johnson, D.S. Computers and Intractability: A Guide to the Theory of NP-Completeness; Freeman: San Francisco, CA, USA, 1979.

14. Bernal Sánchez, J.J. Árboles Romanos. Bachelor's Thesis, Facultad de Ciencias, UNAM, Mexico City, Mexico, 2017. 